\title{
PERSONALITY TRAITS ARE DIFFERENTLY ASSOCIATED WITH DEPRESSION AND ANXIETY: EVIDENCE FROM APPLYING BIVARIATE MULTIPLE BINARY LOGISTIC REGRESSION ON A LARGE SAMPLE OF GENERAL ADULTS
}

\author{
Fatemeh Nouri ${ }^{1,2,3}$, Awat Feizi, ${ }^{2,4,5}$, Ammar Hassanzadeh Keshteli ${ }^{5,6}$, Hamidreza Roohafza ${ }^{7}$, \\ Hamid Afshar ${ }^{7,8}$ \& Peyman Adibi ${ }^{5,9}$ \\ ${ }^{1}$ Hypertension Research Center, Cardiovascular Research Institute, \\ Isfahan University of Medical Sciences, Isfahan, Iran \\ ${ }^{2}$ Department of Biostatistics and Epidemiology, School of Health, Isfahan University of Medical Sciences, Isfahan, Iran \\ ${ }^{3}$ Student Research Committee, School of Health, Isfahan University of Medical Sciences, Isfahan, Iran \\ ${ }^{4}$ Psychosomatic Research Center, Isfahan University of Medical Sciences, Isfahan, Iran \\ ${ }^{5}$ Gastroenterology and Hepatology Research Center, Isfahan University of Medical Sciences, Isfahan, Iran \\ ${ }^{6}$ Department of Medicine, University of Alberta, Alberta, Canada \\ ${ }^{7}$ Cardiac Rehabilitation Research Center, Cardiovascular Research Institute, \\ Isfahan University of Medical Sciences, Isfahan, Iran \\ ${ }^{8}$ Department of Psychiatry, School of Medicine, Isfahan University of Medical Sciences, Isfahan, Iran \\ ${ }^{9}$ Section of Gastroenterology and Hepatology, Department of Internal Medicine, School of Medicine, \\ Isfahan University of Medical Sciences, Isfahan, Iran
}

received: 23.5.2018;

revised: 29.9.2018;

accepted: 5.11 .2018

\section{SUMMARY}

Background: The purpose of this study was to explore the association of five factors personality traits, as predictor variables, with the anxiety and depression as joint dependent variables in an Iranian adult population.

Subjects and methods: A total of 3175 subjects living in Isfahan participated in this cross-sectional population-based "study (SEPAHAN) and completed self-administered questionnaires about demographic, life style, gastrointestinal disorders, personality traits, social support, and psychological problems. Data was analyzed using bivariate multiple binary logistic regression in $R$ Free statistical software.

Results: The results indicated high scores of neuroticisms increase the risk of anxiety and depression after adjustment for the potential confounders in total sample (OR (95\% CI): 1.22 (1.19-1.24) and 1.19 (1.17-1.21), respectively) as well as in both male and female. In contrast, joint inverse associations were found between anxiety and depression with high extraversion (OR (95\% CI): 0.90 (0.88-0.92) and 0.91 (0.89-0.92), respectively), agreeableness (0.93 (0.91-0.96) and 0.94 (0.92-0.96) respectively) and conscientiousness scores (0.95 (0.93-0.97) and 0.95 (0.94-0.97) respectively) as well as in both male and female. Furthermore, higher scores of openness had significant inverse association with depression in male.

Conclusion: The present study indicated that higher scores of neuroticism, however lower extraversion, conscientiousness and agreeableness scores are risk factors for both anxiety and depression in Iranian adult population. It is suggested to perform family studies or twin and genetic association studies with considering combinations of personality traits (personality styles), and also measuring personality traits at the facet level.

Key words: personality traits - anxiety - depression - bivariate binary logistic regression

$$
* * * * *
$$

\section{INTRODUCTION}

Since Hippocrates, who described four types of temperament and related them to both (the lack of) physical and mental health, many theories have been developed to reflect on the association between personality characteristics and psychopathology. Personality traits can be defined as stable patterns of thinking, feelings and behaviors, which are rather consistent during lifetime and can be described according to five broad dimensions, also known as the Big Five personality domains including Neuroticism (easily upset, not calm, and maladjusted), Extraversion (energetic, assertive, and talkative), Conscientiousness (responsible, dependable, and orderly), Openness to Experience (imaginative, independent-minded, and intellectual), and Agreeableness as the opposite of Antagonism (good-natured, cooperative, and trusting) (Koorevaar et al. 2013).

Psychiatric disorder is the major public mental health problem throughout the world that has impact on every aspect of life, including physical health and risk behavior (Prince et al. 2007). Anxiety and depression disorders represent the most prevalent psychiatric disorders, with a considerable burden associated with them, 
not only for individual sufferers, but also for the health care system (Abrignani et al. 2014). Also, mental health problems are responsible for considerable disability worldwide in which it is estimated that by the year 2020, major depression will be second after ischemic heart disease in terms of amount of disability experienced by sufferers (Lecrubier 2001). Therefore, the mental health area constitutes a public health priority.

There are evidences establishing that shared genetic etiologies and personality traits are potential predictors of anxiety and depression and their co morbidities (Hettema 2008). Also, personality structure is considered a significant source of vulnerability for the onset, development, and treatment of various psychiatric conditions (Meuti et al. 2014). Studies show that depression and anxiety scores were significantly and positively correlated with neuroticism scores and were also negatively correlated with extraversion scores both in men and women in the general population (Bienvenu et al. 2001a, Bienvenu et al. 2001b, Newbury-Birch \& Kamali 2001). Lower-order dimensions (facets) of conscientiousness are most frequently low in major depressive and anxiety disorders. Also, lower-order dimensions of agreeableness have been linked to some anxiety disorders (Bienvenu et al. 2004). Also individual with higher neuroticism and lower extraversion scores simultaneously experience higher anxiety scores (Røvik et al. 2007). There is evidence that subjects with current depression have higher mean neuroticism scores than those in remission; those in remission have higher mean neuroticism scores than those with no history of depression (Bienvenu et al. 2001a).

Majority of previous studies on the relationships between personality traits and mental problems, in one hand, have focused only on one problem in relation with only two of the five higher-order factors, i.e. neuroticism and extraversion. Additionally, majority of these studies have been conducted on specific population such as patients and college students so that it is unclear to what extent their findings are applicable to the general population. On the other hand, majority of the conducted previous studies have used simple statistical methods and they did not adjust the impacts of possible confounders.

The main objective of current study was to investigate the relationships between depression and anxiety with five-factor personality traits controlling for the impacts of some important possible confounders including sex, age, marital status, education level, body mass index, social support, smoking behavior, physical activity and some physical illness such as Functional gastrointestinal disorders (FGIDs) using a comprehensive statistical method i.e. bivariate multiple logistic regression in a large sample of Iranian adults. The multivariate or its special future i.e. bivariate logistic regression approach is a simultaneous logistic regression (in the case where scales are dichotomized) for the outcomes (in current study depression and anxiety) predicting by the several predictors in a single analysis. The strengths of the approach include the following: 1) it retains the complete information about case status for each outcome; 2) it permits assessment of outcomes-risk factor interactions as well as "overall" risk factor effects; 3) it provides measures of association between the multiple predictors and adjusts for the association between responses in the analysis.

\section{SUBJECTS AND METHODS}

\section{Study population and setting}

The current study is a part of the "Study on the Epidemiology of Psychological, Alimentary Health and Nutrition" (SEPAHAN) (Adibi et al. 2012). In this cross-sectional study, the studied sample was selected using multistage cluster sampling and convenience sampling in last stage among 4 million people in 20 cities across Isfahan province. In SEPAHAN study, data were collected in two separate phases to increase the accuracy as well as the response rate. In the first phase, all participants were asked to complete a self-administered questionnaire about demographic and lifestyle factors including nutritional habits and dietary intakes. In the second phase, further information on gastrointestinal functional disorders and different aspects of psychological variables were collected using another bunch of self-administered questionnaires (response rate: 86.16\%). In the current analysis, we used data from 3175 adults who had completed data on demographic data, personality traits, social support, and psychological outcomes such as depression and anxiety. The study protocol was clarified and a written informed consent was obtained from all participants. The ethics committee of Isfahan University of Medical Sciences approved the study.

\section{Assessment of psychological variables Hospital Anxiety and Depression Scale (HADS)}

HADS records levels of depression and anxiety symptoms. Seven items assess levels of anxiety symptoms and seven ones assess levels of depression symptoms, in a four-point scale. The sum score of each subscale (levels of anxiety and depression symptoms) ranges from 0 to 21 . The recommended cut-off score $\geq 8$ indicates a possible major depression or clinical anxiety disorder. Internal consistency measured by Cronbach's alpha has been found to be 0.78 and 0.86 for HADS anxiety and depression sub-scale respectively in Iranian population (Montazeri et al. 2003).

\section{Assessment of personality traits}

Among available instruments for measuring five factors, the NEO Five-Factor Inventory (NEO-FFI) and the NEO Personality Inventory Revised (NEO-PI-R) are the most widely used. NEO-FFI was a shortened version of the NEO-PI-R (240-items). The NEO-FFI consists of 60 self-descriptive statements about the personality that measuring five dimensions of the normal personality (i.e. neuroticism $(\mathrm{N})$, extraversion $(\mathrm{E})$, openness $(\mathrm{O})$, 
agreeableness (A) and conscientiousness (C)) and consisting 12 item per dimension. Respondents indicate the degree to which they agree or disagree with each of the statement using a five-point Likert-type scale $(0=$ strongly disagree, $4=$ strongly agree $)$. Eight items required reverse scoring. Individual subscale values were determined by summing the 12 items (Afshar et al. 2015). In Iranian population, Cronbach's alpha was shown for $\mathrm{N}, \mathrm{E}, \mathrm{O}, \mathrm{A}$, and $\mathrm{C}$ as $0.76,0.65,0.59,0.48$ and 0.75 , respectively (Atari et al. 2006).

\section{Assessment of other variables}

Self-administered standard questionnaires were distributed to collect information on age (years), gender (male/female), marital status (married, single), selfreported weight $(\mathrm{kg})$, height $(\mathrm{cm})$, smoking (none, former and current smokers). Body mass index (BMI) was calculated by dividing weight in kilograms by height in meters squared. Educational attainments categorized into three categories i.e. lower than diploma (12 years formal education), diploma and university graduation (including bachelor, master and doctorate). Self-reported history of major FGIDs including Gastro Esophageal acid Reflux Disease (GERD), functional dyspepsia (FD), Functional constipation (FC) and irritable bowel Syndrome (IBS) was explored. The Rome III questionnaire in its complete form and additional questions from the Talley Bowel Disease Questionnaire were used to diagnose and classify FGIDs. Face validation of this questionnaire indicated that most participants could not discriminate the difference between the rating scales used in Rome III. Therefore, rating scales were modified to a 4 -item-rating scale (never or rarely, sometimes, often, always) for each question. Details of some changes in Rome III were described in former publications (Adibi et al. 2012). In the current study, the number of FGIDs was considered (Ranging from 0 to 4). Perceived social support was measured using Multidimensional Scale of Perceived Social Support (MSPSS) that consisted of 12 specific questions and 3 sources of support: family, friends, and significant other (Zimet et al. 1988). In current study the rescoring form of MSPSS has been used; in which each item scored from 0 (disagree and neutral) to 1 (agree), led to a total score between 0 and 12. Higher score represents higher social support. Validity and reliability of the scale has been evaluated in Iran (Ghaedi \& Yaaghoobi 2008). General Practice Physical Activity Questionnaire (GPPAQ) was used to assess an individual's current physical activity status. The GPPAQ consist of work and leisure time physical activity. It generates simple, 4-level Physical Activity Index (PAI) categorizing subjects as: Active, Moderately Active, Moderately Inactive, and Inactive (Adibi et al. 2012). In the current analysis, participants were classified into two categories namely inactive (including inactive and moderately inactive) and active (including moderately active and active as earlier indicates).

\section{Statistical analysis}

Data were analyzed by R Free Statistical Software version 3.1.1. Results were presented as mean \pm standard deviation (SD) for quantitative variables while frequencies and percentages for qualitative ones. T-test or Mann-Whitney U test (as appropriate) were used to compare mean differences of quantitative variables between two groups. Distribution of study participants in terms of qualitative variables across different categories of other variables was compared using chi-square test. Bivariate multiple binary logistic regression model was performed for joint modeling of anxiety and depression as dichotomous dependent variables and personality traits as independent variables. Adjusted odds ratio (OR) and $95 \%$ confidence intervals (CI) for the association of personality traits with affecting by depression and anxiety are presented in 4 different models. First, adjustment was made for demographic variables including age, sex, marital status, educational levels. We further controlled the confounding impacts of lifestyle variables including smoking, BMI, physical activity in the second model. Additional adjustments were made for number of FGIDs in third model. In the final model, a further adjustment was made for social support. In all models, non-anxious and non-depressed people were considered as the reference categories. p-value less than 0.05 were considered statistically significant.

\section{RESULTS}

The mean (SD) age of participants was $35.91(7.76)$ years. $60.5 \%$ of sample was female and $61.4 \%$ had college education. About $16 \%$ of subjects were current or ex-smokers. $46.5 \%$ of study subjects participated in regular physical activity. About $53 \%$ of the respondents reported experiencing at least one of major FGIDs including GERD, FD, FC and IBS. Prevalence of anxiety and depression were $14.1 \%$ and $28.9 \%$, respectively.

Prevalence of anxiety and depression in different categories of gender, marital status, education levels, smoking, physical activity and number of FGIDs are presented in Table 1. The percentages of women with anxiety $(17.2 \%$ versus $9.5 \%(\mathrm{P}<0.001))$ and depression $(33.7 \%$ versus $21.6 \% \quad(p<0.001))$ were significantly higher than men. The prevalence of anxiety was $22.3 \%$, $17.8 \%$ and $11.0 \%$ in individuals with $0-12,12$ and more than 12 years of education, respectively $(\mathrm{P}<0.001)$. The prevalence of anxiety and depression was significantly higher in inactive participants than active ones (for both $\mathrm{P}<0.001)$. The higher suffering from different FGIDs was associated with the higher prevalence of anxiety and depression, $5.6 \%$ to $44.4 \%$ for anxiety, and $17.3 \%$ to $55.6 \%$ for depression across different number of FGIDs (0-4) respectively $(\mathrm{P}<0.001)$. Also, participants with anxiety and depression were more likely to report a lack of social supports $(\mathrm{P}<0.001)$. 


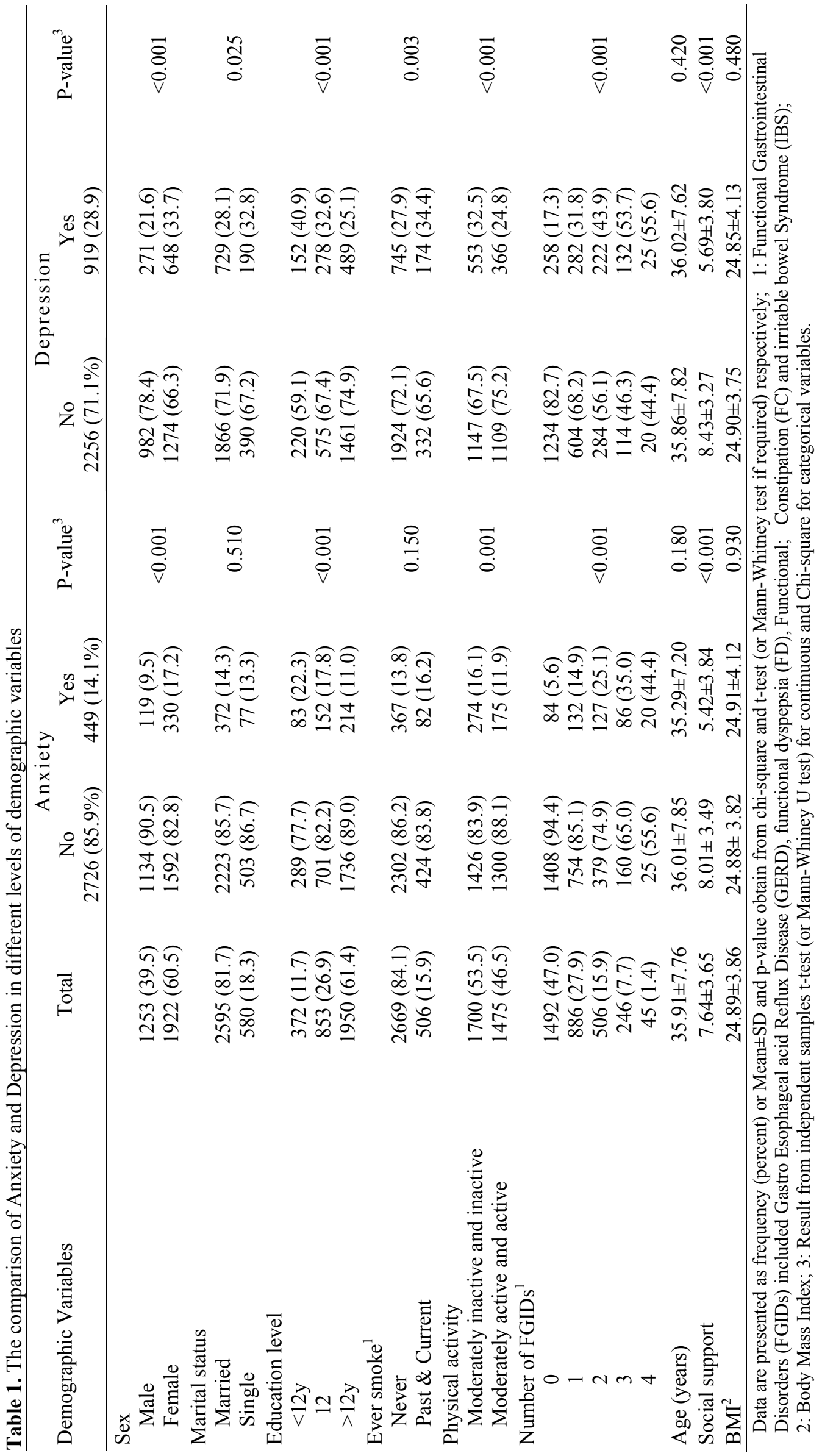


Table 2. Crude and adjusted odds ratio (OR; 95\% CI for ORs) resulted from joint modeling of anxiety and depression level as dependent variables and five factors personality traits as independent variables

\begin{tabular}{|c|c|c|c|c|c|}
\hline \multirow{2}{*}{$\begin{array}{l}\text { Independent } \\
\text { variables }\end{array}$} & \multicolumn{5}{|c|}{ Dependent variables } \\
\hline & Crude & Model 1 & Model 2 & Model 3 & Model 4 \\
\hline \multicolumn{6}{|l|}{ ANXIETY } \\
\hline \multicolumn{6}{|l|}{ Female } \\
\hline Neuroticism & $1.24(1.21-1.27)$ & $1.24(1.21-1.27)$ & $1.24(1.21-1.27)$ & $1.23(1.19-1.26)$ & $1.22(1.19-1.25)$ \\
\hline Extraversion & $0.88(0.86-0.89)$ & $0.88(0.86-0.90)$ & $0.88(0.87-0.90)$ & $0.89(0.87-0.91)$ & $0.90(0.88-0.92)$ \\
\hline Openness & $0.98(0.96-1.01)$ & $1.001(0.97-1.03)$ & $1.003(0.98-1.03)$ & $0.99(0.97-1.02)$ & $1.01(0.99-1.04)$ \\
\hline Agreeableness & $0.91(0.89-0.93)$ & $0.92(0.90-0.94)$ & $0.92(0.89-0.94)$ & $0.92(0.90-0.94)$ & $0.93(0.91-0.96)$ \\
\hline Conscientiousness & $0.93(0.91-0.95)$ & $0.93(0.92-0.95)$ & $0.93(0.92-0.95)$ & $0.94(0.92-0.96)$ & $0.95(0.93-0.97)$ \\
\hline \multicolumn{6}{|l|}{ Male } \\
\hline Neuroticism & $1.25(1.21-1.29)$ & $1.25(1.21-1.29)$ & $1.25(1.20-1.29)$ & $1.23(1.18-1.27)$ & $1.21(1.16-1.27)$ \\
\hline Extraversion & $0.87(0.84-0.89)$ & $0.87(0.84-0.89)$ & $0.87(0.84-0.89)$ & $0.89(0.86-0.92)$ & $0.91(0.88-0.95)$ \\
\hline Openness & $0.99(0.95-1.03)$ & $0.99(0.95-1.03)$ & $0.99(0.95-1.03)$ & $0.99(0.95-1.04)$ & $1.02(0.97-1.07)$ \\
\hline Agreeableness & $0.89(0.87-0.92)$ & $0.89(0.87-0.93)$ & $0.89(0.87-0.93)$ & $0.92(0.89-0.95)$ & $0.94(0.90-0.97)$ \\
\hline Conscientiousness & $0.92(0.89-0.95)$ & $0.92(0.89-0.95)$ & $0.92(0.89-0.95)$ & $0.95(0.92-0.98)$ & $0.97(0.93-0.99)$ \\
\hline \multicolumn{6}{|l|}{ Total } \\
\hline Neuroticism & $1.25(1.22-1.27)$ & $1.24(1.22-1.27)$ & $1.24(1.22-1.27)$ & $1.23(1.20-1.25)$ & $1.22(1.19-1.24)$ \\
\hline Extraversion & $0.87(0.86-0.89)$ & $0.88(0.86-0.89)$ & $0.88(0.86-0.89)$ & $0.88(0.87-0.90)$ & $0.90(0.89-0.92)$ \\
\hline Openness & $0.99(0.96-1.01)$ & $0.99(0.98-1.02)$ & $0.99(0.98-1.02)$ & $0.99(0.97-1.02)$ & $1.01(0.99-1.04)$ \\
\hline Agreeableness & $0.91(0.89-0.93)$ & $0.91(0.89-0.93)$ & $0.91(0.89-0.93)$ & $0.92(0.90-0.94)$ & $0.93(0.92-0.95)$ \\
\hline Conscientiousness & $0.93(0.91-0.94)$ & $0.93(0.91-0.94)$ & $0.93(0.91-0.95)$ & $0.94(0.92-0.95)$ & $0.95(0.94-0.97)$ \\
\hline
\end{tabular}

\section{DEPRESSION}

\begin{tabular}{|c|c|c|}
\hline \multicolumn{3}{|l|}{ Female } \\
\hline Neuroticism & $1.21(1.19-1.24)$ & 1.21 (1.19- \\
\hline Extraversion & $0.87(0.86-0.89)$ & $0.88(0.86-$ \\
\hline Openness & $0.97(0.95-0.98)$ & $0.98(0.96$ \\
\hline Agreeableness & $0.91(0.89-0.93)$ & $0.92(0.90$ \\
\hline Conscientiousness & $0.93(0.92-0.95)$ & $0.93(0.92$ \\
\hline \multicolumn{3}{|l|}{ Male } \\
\hline Neuroticism & $1.24(1.21-1.28)$ & $1.24(1.21$ \\
\hline Extraversion & $0.85(0.83-0.88)$ & $0.85(0.83$ \\
\hline Openness & $0.94(0.91-0.97)$ & $0.95(0.92$ \\
\hline Agreeableness & $0.89(0.87-0.91)$ & $0.89(0.87$ \\
\hline Conscientiousness & $0.91(0.89-0.93)$ & $0.90(0.88$ \\
\hline \multicolumn{3}{|l|}{ Total } \\
\hline Neuroticism & $1.23(1.21-1.25)$ & $1.22(1.20$ \\
\hline Extraversion & $0.86(0.85-0.88)$ & $0.87(0.86$ \\
\hline Openness & $0.96(0.95-0.98)$ & $0.97(0.95$ \\
\hline Agreeableness & $0.91(0.89-0.93)$ & $0.91(0.89$ \\
\hline Conscientiousness & $0.92(0.91-0.94)$ & $0.92(0.91$ \\
\hline \multicolumn{3}{|c|}{$\begin{array}{l}\text { Comparisons of mean scores of five domains of } \\
\text { personality traits between anxious and non-anxious as } \\
\text { well as depressed and non-depressed participants were } \\
\text { provided in Figure } 1 \text { and } 2 \text {, respectively. The mean scores } \\
\text { of neuroticisms were statistically higher in anxious and } \\
\text { depress people than healthy ones }(\mathrm{P}<0.001) \text {. In contrast, } \\
\text { participants with anxiety and depression were more likely } \\
\text { to have lower extraversion, agreeableness, conscientious- } \\
\text { ness and openness (except for anxiety) scores than those } \\
\text { without anxiety and depression }(\mathrm{P}<0.001) \text {. }\end{array}$} \\
\hline
\end{tabular}

Female

Neuroticism

Openness

Agreeableness ale

Neuroticism

Agreeableness Col

Neuroticism

Extraversion

Openness

Agreeableness

$0.91(0.89-0.93)$

92)

$$
\text { 4) } 1.2
$$

$1.21(1.19-1.24)$

$0.88(0.86-0.89)$

$0.98(0.96-1.002)$

$0.92(0.90-0.94)$

$0.93(0.92-0.95)$

$1.20(1.18-1.23)$
$0.88(0.87-0.89)$
$0.98(0.96-0.99)$
$0.92(0.91-0.94)$
$0.94(0.92-0.95)$

$1.19(1.16-1.21)$

$0.91(0.89-0.92)$

$0.99(0.97-1.01)$

$0.94(0.92-0.96)$

$0.95(0.94-0.97)$
$1.24(1.21-1.28) \quad 1.23(1.19-1.27) \quad 1.21(1.17-1.25)$
$0.86(0.83-0.88) \quad 0.87(0.85-0.89) \quad 0.88(0.86-0.91)$
$0.94(0.91-0.97) \quad 0.94(0.91-0.97) \quad 0.96(0.93-0.99)$
$0.89(0.87-0.93) \quad 0.90(0.88-0.93) \quad 0.92(0.89-0.95)$
$0.91(0.89-0.93) \quad 0.92(0.89-0.94) \quad 0.94(0.92-0.96)$

$1.22(1.20-1.24) \quad 1.21(1.19-1.23) \quad 1.19(1.17-1.21)$

$0.87(0.86-0.88) \quad 0.88(0.86-0.89) \quad 0.90(0.88-0.91)$

$0.97(0.95-0.99) \quad 0.97(0.95-0.99) \quad 0.98(0.97-1.001)$

$0.91(0.89-0.92) \quad 0.91(0.90-0.93) \quad 0.93(0.92-0.95)$

$0.93(0.91-0.94) \quad 0.93(0.92-0.95) \quad 0.95(0.93-0.96)$

\begin{abstract}
well as depressed and non-depressed participants were provided in Figure 1 and 2, respectively. The mean scores of neuroticisms were statistically higher in anxious and depress people than healthy ones $(\mathrm{P}<0.001)$. In contrast, participants with anxiety and depression were more likely ness and openness (except for anxiety) scores than those without anxiety and depression $(\mathrm{P}<0.001)$.
\end{abstract}

Comparisons of mean scores of five domains of
Joint modeling of anxiety and depression (as dichotomous response variables) on the personality traits as the predictor variables in total sample as well as in gender categories (stratified analyses by gender) in different models were given in Table 2. Considering joint modeling of anxiety and depression provides the possibility for accounting correlation and co-morbidity between these outcomes. In all fitted models, those participants without depression and anxiety were considered as the reference category. 


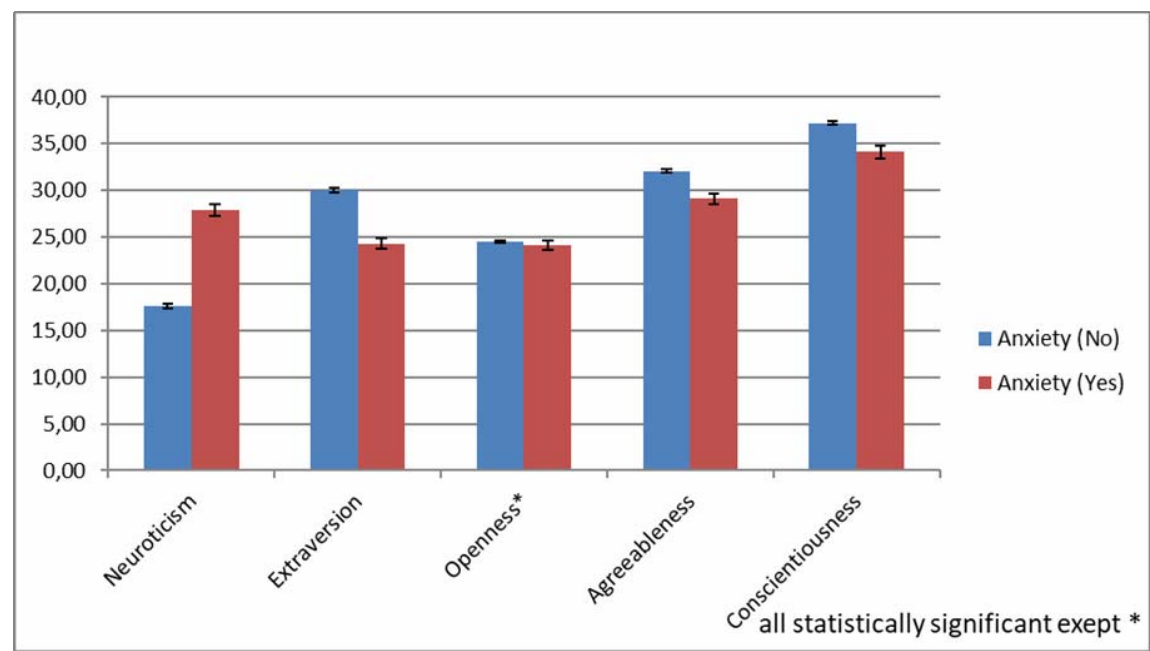

Figure 1. Comparison of mean scores of five domains of personality traits between anxious and non-anxious participants

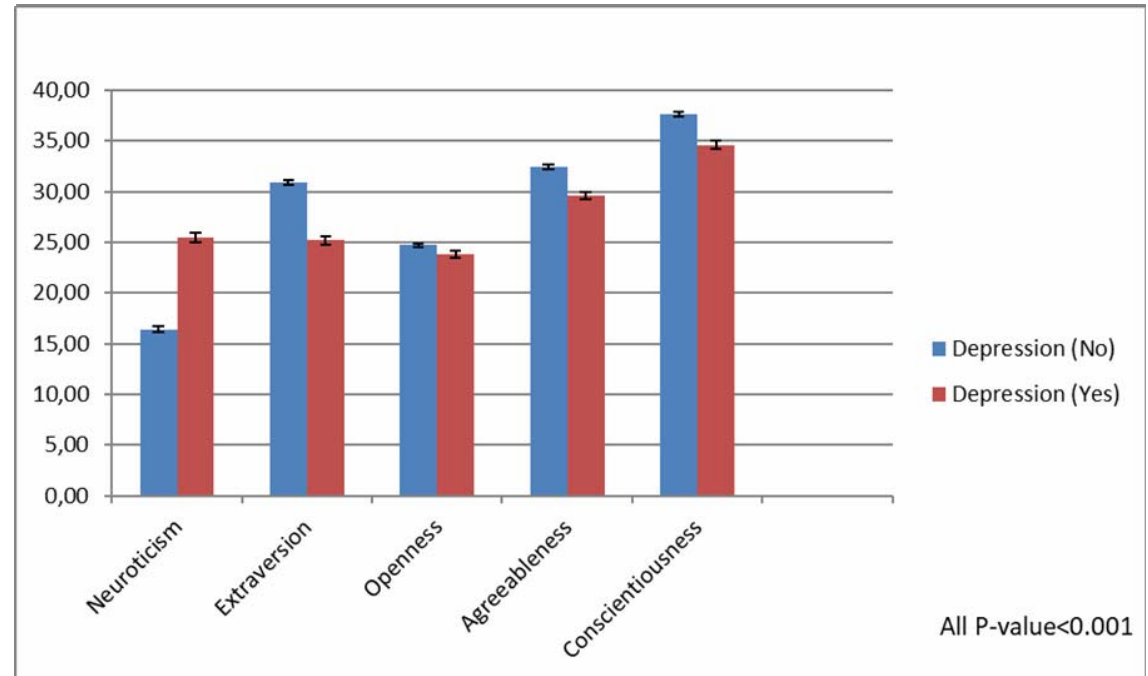

Figure 2. Comparisons of mean scores of five domains of personality traits between depressed and non-depressed participants

In crude models, we reached a joint significant positive association between anxiety and depression with neuroticism scores (OR: 1.25; 95\% CI: 1.22-1.27 and OR: 1.23 ; 95\% CI: $1.21-1.25$, respectively). Also, the sex-based association between the odds of having anxiety and depression with personality traits are shown in Table 2. The ORs in the crude models were $1.24(95 \% \mathrm{CI}$ : $1.21-1.27)$ and 1.25 (95\% CI: 1.21-1.29) for anxiety and 1.21 (95\%CI 1.19-1.24) and 1.24 (95\% CI 1.21-1.28) for depression in female and male, respectively. On the contrary, we found joint inverse associations between anxiety and depression with higher extraversion scores (OR: 0.87; 95\% CI: 086-0.89 and OR: 0.86 ; 95\% CI: $0.85-0.88$, respectively) as well as in male and female. Likewise, negative significant relationships were found between studied psychological disorders and high-agreeableness and conscientiousness scores both in total sample as well as in male and female participants. Furthermore, there were inverse significant associations between depression and high openness score (OR: 0.96; 95\% CI: $0.95-0.98)$, but no significant association between openness scores and anxiety in crude model.
Adjusted odds ratio (OR) and 95\% confidence intervals (CI) for depression and anxiety are presented in 4 different models. After adjusting for a wide range of potential confounding variables such as age, sex, marital status, education level, ever smoke, BMI, physical activity, number of FIGDs and social supports, the same findings were also found about the association of depression and anxiety with neuroticism, extraversion, agreeableness and conscientiousness scores in male, female and total population.

The ORs of neuroticism score in the final fully adjusted bivariate multiple binary logistic regression in total population, were 1.22 (95\% CI: $1.19-1.24)$ for anxiety and 1.19 (95\% CI: 1.17-1.21) for depression. Also, the ORs of the association of neuroticism with anxiety and depression were 1.22 (95\%CI: $1.19-1.25)$ and 1.21 (95\% CI: $1.16-1.27)$ in male and 1.19 (95\% CI 1.16-1.21) and 1.21 (95\% CI 1.17-1.25) in female, respectively. On the contrary, the chance of anxiety and depression was significantly decreased with increasing extraversion, agreeableness and conscientiousness scores of personality traits. The significant protective roles 
were found for extraversion (OR: 0.90) and (OR: 0.91); for agreeableness (OR: 0.93) and (OR: 0.94); for conscientiousness (OR: 0.95) and (OR: 0.95) after adjustment for all considered confounders in total, on anxiety and depression, respectively as well as in both male and female. As Table 2 shows, we reached no significant association between the two studied psychological disorders and openness in fully adjusted except for depression in male (OR: 0.96; 95\% CI: 0.93-0.99). More details on the relationship between anxiety and depression with personality traits in different models based on sex can be found in Table 2 .

\section{DISCUSSION}

Joint modeling of anxiety and depression is an efficient approach for considering their comorbidity and co-occurring. In this cross-sectional population-based study, the significant association between five -factor personality traits and common psychological disorders (anxiety and depression) was supported by the data from a large sample of Iranian adults' population.

Current study concluded that the higher scores of neuroticisms, after controlling for the impacts of major potential confounders such as age, sex, marital status, education level, smoking, BMI, physical activity, number of FGIDs and social supports "(fully adjusted model)", was simultaneously associated with increasing risk of anxiety and depression in separately fitted models for male and female samples as well for total population. The evidence from prospective association of neuroticism with common mental disorders has strengthen the assumption that the neuroticism is an independent etiologically risk factor. Neuroticism also plays an important role in other phenomena that correlate strongly with other psychological affectivity such as persistent low subjective well-being, and physical health problems. Neuroticism accounts for a substantial proportion of current and lifetime comorbidity, most strongly within the domain of internalizing disorders, but also between internalizing and externalizing problems and between mental and physical disorders. A comprehensive literatures review indicates that (1) neuroticism predicts onset of common mental disorders even after controlling for most (but not all) psychiatric confounders; (2) items used to assess neuroticism partially overlap with mental disorders symptoms, especially for internalizing disorders; (3) neuroticism and mental disorders share substantial but not all genetic and environmental determinants; (4) neuroticism has higher temporal stability than mental disorders symptoms, although the difference is smaller than commonly thought; (5) neuroticism probably moderates the impact of life stress on mental disorders; and (6) reductions in neuroticism may partially mediate the effect of treatment on mental disorders (Ormel et al. 2013).

Our study results indicated that the high scores of extraversion, agreeableness and conscientiousness, after controlling for the impacts of all possible confounding variables in the fully adjusted model, decreased the risk of anxiety and depression. Although, the openness showed protective role regarding suffering from anxiety and depression, however the associations were not statistically significant in fully adjusted models in total, female and male samples. Extraversion, agreeableness and openness are related to positive emotionally dimensions of five factors model and there is some evidence that positive affect moderates the effects of daily stressors on depressive and anxiety symptoms. Although, we observed an inverse association between conscientiousness with anxiety and depression, however the previous studies showed that it has reciprocal impacts on family supports, divorce, occupational attainment, and job involvement. Conscientiousness is hypothesized to affect depression by increasing exposure to negative life events but mediation and moderation effects have not been tested (Klein et al. 2011).

Similar results were observed in many previous studies. Newbury-Birch and Kamali (2001) observed a significant inverse relationship between neuroticism and both depression and anxiety without adjustment for confounding variables in male and female. This study further revealed that women who had lower extraversion scores had higher anxiety and depression scores (Newbury-Birch \& Kamali 2001). In another study, by applying binary logistic regression it was found that greater scores of neuroticisms were significantly associated with increased risk of affecting by depression. They also showed that the extraversion (and facets of assertiveness, activity, and positive emotionality) and conscientiousness (and facets of competence, order, dutifulness, and self-discipline) are associated with reduced risk of depression (Hayward et al. 2013).

Bienvenu et al. (2004) demonstrated that anxiety and depression disorders were associated with higher neuroticism scores and some anxiety disorders were associated with lower extraversion scores. They also showed that the agreeableness and conscientiousness were inversely associated with some specific anxiety disorders. The factor- and facet-level personality profiles are significantly different between subjects suffering from each of the lifetime anxiety or depressive disorders and non-affected subjects (Bienvenu et al. 2004).

Wang et al. (2014) through fitting a hierarchical linear regression analysis, after adjusting the confounding impacts of potential confounders, demonstrated that extraversion, agreeableness and conscientiousness scores were negatively associated with depressive symptoms whereas neuroticism was positively associated with (Wang et al. 2014). In another study, based on fitting multiple linear regressions, it was shown that the depression severity and age of depression onset were significantly associated with higher levels of neuroticism and lower levels of extraversion and conscientiousness. Also earlier onset of depression was significantly associated with higher levels of openness (Koorevaar et al. 2013). In another study, among general population 
by using multivariate analysis of variance it was indicated that neuroticism, extraversion, and facets of agreeableness and conscientiousness play significant role in understanding the depressive and anxiety conditions. Neuroticism is broadly associated with social phobia, agoraphobia, panic disorder, and major depression. introversion is only broadly associated with social phobia and agoraphobia. Lower order dimensions of agreeableness appear to be related to phobias, and conscientiousness to phobic, panic and major depressive disorders (Bienvenu et al. 2001b).

Kotov et al. (2010) performed a quantitative review about the associations between personality traits in the Big Three and Big Five models and specific depressive, and anxiety symptoms in adults. They found higher scores of neuroticism and lower scores of conscientiousness in people diagnosed with psychological disorders. Although, they found that the agreeableness and openness were not notably related to those studied disorders; however they generally concluded that common mental disorders are strongly linked to personality and have similar trait profiles (Kotov et al. 2010).

It is suggested to perform family studies demonstrating personality differences between non affected relatives of probands with and without a history of psychiatric disorders and twin and genetic association studies demonstrating the same genes predispose to both personality and psychiatric disorders (Kendler et al. 2006, Kendler et al. 1993, Klein et al. 2011). Also, it seems that facet-level research promises to yield stronger and more specific evidence about personality-psychological disorders profile links. Measuring personality traits at the facet level captures aspects of the relationship between personality and psychological diseases that are overlooked when taking an approach based on personality factors alone (Hayward et al. 2013). Some evidences highlight the importance of examining combinations of personality traits or personality styles when identifying those who are most at-risk of psychological disorders. The analysis of personality styles is consistent with the idea that high and low levels of traits can be pathogenic, and that all traits have adaptive and maladaptive consequences (Weiss et al. 2009).

Higher rates of psychiatric comorbidity as well as more impaired psychosocial adjustment occur with the functional gastrointestinal disorders. Inversely, some studies show higher rates of functional gastrointestinal disorders with psychiatric diagnoses. Addressing coexisting of psychiatric illness with functional bowel disturbances will improve treatment outcome. On the other hand, psychological factors affect the clinical expression of structural disease (Shah et al. 2014). There is a bidirectional association between functional gastrointestinal disorders and psychiatric illness accordingly it is suggested to study the mediating effects of gastrointestinal disorders on the association analyses between personality traits and psychological disorders through another statistical modeling approach such structural equation models.

\section{Study strengths and limitations}

Some strengths of current study included the use of an advanced statistical method that provide the possibility for simultaneous modeling of anxiety and depression risk considering the comorbidity and cooccurrence between them and considering five personality traits as the predictors in all fitted models. On the other hand, the impacts of wide range of potential confounding variables, including sex, age, marital status, education level, body mass index, social supports, smoking, physical activity and number of FGIDs have been adjusted for estimating the association of personality traits and studied psychological disorders. Large and representative sample from a general population have been investigated.

However, our study has several limitations: most important among them, first, due to the cross-sectional design, no causal inferences can be drawn. Second the study's results are based on participants' self-ratings, lead to subjective responses. In this regard, it would be particularly informative to assess such variables using multiple methods (e.g., self-ratings and clinicians' ratings). This multi-method design would permit powerful analyses of personality-psychopathology relations. Furthermore, we adopted a simple approach to handle limited available missing data (i.e. complete-case) as our data were fairly complete. However, future studies may benefit from more sophisticated methods, such as multiple imputations.

\section{CONCLUSIONS}

The present study indicated that high neuroticism, low extraversion, conscientiousness and agreeableness scores were linked to increase the anxiety and depression in Iranian adult population. It is suggested to perform family studies or twin and genetic association studies with considering combinations of personality traits (personality styles), and also measuring personality traits at the facet level. Investigation of relations between personality traits and mental health problems can provide important information about the dimensional measures of psychopathology and screening for psychological factors in the primary care settings.

\section{Contribution of individual authors:}

Fatemeh Nouri contributed to statistical analysis. Awat Feizi supervised the current secondary study.

Fatemeh Nouri \& Awat Feizi contributed to results interpretation and manuscript drafting.

Ammar Hassanzadeh Keshteli, Hamidreza Roohafza, Hamid Afshar \& Peyman Adibi contributed to SEPAHAN study (main study)'s design, data collection and drafting the current secondary study.

All authors read and approved the final version of submitted manuscript. 


\section{Acknowledgements:}

The authors are thankful to participants of SEPAHAN project, the current study article was a research project at Isfahan University of Medical Sciences, under project number: 194130 .

\section{Conflict of interest: None to declare.}

\section{References}

1. Abrignani $M G$, Renda $N$, Abrignani $V$, Raffa A, Novo $S$ \& Baido RL: Panic disorder, anxiety, and cardiovascular diseases. Clin Neuropsychiatry 2014; 11:130-144

2. Adibi P, Keshteli AH, Esmaillzadeh A, Afshar H, Roohafza H, Bagherian-Sararoudi $R$ et al.: The study on the epidemiology of psychological, alimentary health and nutrition (SEPAHAN): Overview of methodology. J Res Med Sci 2012; 17

3. Afshar H, Roohafza HR, Keshteli AH, Mazaheri M, Feizi $A \&$ Adibi P: The association of personality traits and coping styles according to stress level. J Res Med Sci $2015 ; 20: 353$

4. Atari YA, Aman Elahifard A \& Mehrabizadeh HM: An investigation of relationships between ersonality characteristics and family-personal factors and marital satisfaction in administrative office personnel in Ahvaz. J Educ Psychol 2006; 13:81-108

5. Bienvenu OJ, Brown C, Samuels JF, Liang K-Y, Costa PT, Eaton WW et al.: Normal personality traits and comorbidity among phobic, panic and major depressive disorders. Psychiatry Res 2001a; 102:73-85

6. Bienvenu OJ, Nestadt G, Samuels JF, Costa PT, Howard WT \& Eaton WW: Phobic, panic, and major depressive disorders and the five-factor model of personality. J Nerv Ment Dis 2001b; 189:154-161

7. Bienvenu OJ, Samuels JF, Costa PT, Reti IM, Eaton WW \& Nestadt G: Anxiety and depressive disorders and the five factor model of personality: A higher and lower order personality trait investigation in a community sample. Depress Anxiety 2004; 20:92-97. doi:10.1002/da.20026

8. Ghaedi $G$ \& Yaaghoobi H: A study on the Relationship between Different Dimensions of Perceived Social Support and Different Aspects of Wellbeing. Armaghane danesh 2008; 13:69-81

9. Hayward RD, Taylor WD, Smoski MJ, Steffens DC \& Payne ME: Association of five-factor model personality domains and facets with presence, onset, and treatment outcomes of major depression in older adults. Am J Geriatr Psychiatry 2013; 21:88-96

10. Hettema JM: What is the genetic relationship between anxiety and depression? Am J Med Genet C Semin Med Genet 2008; 148:140-146

11. Kendler KS, Gatz M, Gardner CO \& Pedersen NL: Personality and major depression: a Swedish longitudinal, populationbased twin study. Arch Gen Psychiatry 2006; 63:1113-1120
12. Kendler KS, Neale MC, Kessler RC, Heath AC \& Eaves LJ: A longitudinal twin study of personality and major depression in women. Arch Gen Psychiatry 1993; 50:853-862

13. Klein DN, Kotov $R$ \& Bufferd SJ: Personality and depression: explanatory models and review of the evidence. Annu Rev Clin Psychol 2011; 7:269-295

14. Koorevaar A, Comijs H, Dhondt A, van Marwijk H, Van Der Mast R, Naarding $P$ et al.: Big Five personality and depression diagnosis, severity and age of onset in older adults. J Affect Disord 2013; 151:178-185

15. Kotov R, Gamez W, Schmidt F \& Watson D: Linking "big" personality traits to anxiety, depressive, and substance use disorders: A meta-analysis. Psychological Bulletin 2010; 136:768-821

16. Lecrubier Y: The burden of depression and anxiety in general medicine. J Clin Psychiatry 2001; 62(suppl. 8):4-9

17. Meuti V, Marini I, Grillo A, Lauriola M, Leone C, Giacchetti $N$ et al.: MMPI-2: cluster analysis of personality profiles in perinatal depression - preliminary evidence. Scientific World Journal 2014; 2014:964210

18. Montazeri A, Vahdaninia M, Ebrahimi $M$ \& Jarvandi $S$ : The Hospital Anxiety and Depression Scale (HADS): translation and validation study of the Iranian version. Health Qual Life Outcomes 2003; 1:14

19. Newbury-Birch D \& Kamali F: Psychological stress, anxiety, depression, job satisfaction, and personality characteristics in preregistration house officers. Postgrad Med J 2001; 77:109-111

20. Ormel J, Jeronimus BF, Kotov R, Riese H, Bos EH, Hankin $B$ et al.: Neuroticism and common mental disorders: meaning and utility of a complex relationship. Clin Psychol Rev 2013; 33:686-697. doi:10.1016/j.cpr.2013.04.003

21. Prince M, Patel V, Saxena S, Maj M, Maselko J, Phillips MR et al.: No health without mental health. lancet 2007; 370:859-877

22. Røvik JO, Tyssen R, Gude T, Moum T, Ekeberg $\varnothing \&$ Vaglum P: Exploring the interplay between personality dimensions: A comparison of the typological and the dimensional approach in stress research. Pers Individ Dif 2007; 42:1255-1266

23. Shah E, Rezaie A, Riddle M \& Pimentel M: Psychological disorders in gastrointestinal disease: epiphenomenon, cause or consequence? Ann Gastroenterol 2014; 27:224-230

24. Wang Y, Yao L, Liu L, Yang X, Wu H, Wang J et al.: The mediating role of self-efficacy in the relationship between Big five personality and depressive symptoms among Chinese unemployed population: a cross-sectional study. BMC psychiatry 2014; 14:61

25. Weiss A, Sutin AR, Duberstein PR, Friedman B, Bagby $R$ $M \&$ Costa PT: The personality domains and styles of the five-factor model are related to incident depression in Medicare recipients aged 65 to 100. Am J Geriatr Psychiatry 2009; 17:591-601

26. Zimet GD, Dahlem NW, Zimet SG \& Farley GK: The multidimensional scale of perceived social support. J Pers Assess 1988; 52:30-41

\section{Correspondence:}

Awat Feizi, PhD

Department of Biostatistics and Epidemiology,

School of Health, Isfahan University of Medical Sciences

Isfahan, Iran

E-mail:awat_feiz@hlth.mui.ac.ir 